\title{
A review of the deterministic and diffusion approximations for stochastic chemical reaction networks*
}

\author{
Pavel Mozgunov $\dagger$ Marco Beccuti $\ddagger$ Andras Horvath $\ddagger$ Thomas Jaki* \\ Roberta Sirovich\$, Enrico Bibbona
}

\begin{abstract}
This work reviews deterministic and diffusion approximations of the stochastic chemical reaction networks and explains their applications. We discuss the added value the diffusion approximation provides for systems with different phenomena, such as a deficiency and a bistability. It is advocated that the diffusion approximation can be considered as an alternative theoretical approach to study the reaction networks rather than a simulation shortcut. We discuss two examples in which the diffusion approximation is able to catch qualitative properties of reaction networks that the deterministic model misses. We provide an explicit construction of the original process and the diffusion approximation such that the distance between their trajectories is controlled and demonstrate this construction for the examples. We also discuss the limitations and potential directions of the developments.
\end{abstract}

Keywords: Bistable Systems; Deficiency; Diffusion Approximation; Hungarian Construction; Reaction Networks; Stochastic Differential Equations.

\section{Introduction}

A mathematical modelling of chemical kinetics was initiated at the beginning of the previous century. This topic has attracted an extensive attention and works of many excellent scientists formed the Reaction Network Theory at the end of the 1980s. In this formalization, the concentration of species in the network of chemical reactions obeys deterministic laws which are encoded into systems of the non-linear ordinary differential equations (ODEs). These equations provided a rich collection of complex examples and helped to improve the theory of dynamical systems (Érdi and Tóth, 1989; Feinberg, 1972).

Despite the fact that the deterministic models have been sufficient for the majority of applications available at that time, it was already known that a microscopic description of chemical

${ }^{*}$ This is an accepted version of the paper published in Reaction Kinetics, Mechanisms and Catalysis; https: //doi.org/10. 1007/s11144-018-1351-y

'Medical and Pharmaceutical Statistics Research Unit, Department of Mathematics and Statistics, Lancaster University

${ }^{\ddagger}$ Dipartimento di Informatica, Università di Torino

$\S$ Dipartimento di Matematica G. Peano, Università di Torino

^DISMA, Politecnico di Torino 
kinetics should have included randomness (Érdi and Lente, 2014) for historical remarks). The most popular way to describe the stochastic models of reaction networks is in terms of Continuous Time Markov Chain (CTMC). The reactions are considered as happening at random events that modify the state of the network according to the stoichiometric equations. For some time these two descriptions have been developed in parallel and using different tools: the deterministic models were investigated in the theoretical and mathematical aspects, while the stochastic models were mainly studied from a computational point, e.g. in the search of suitable simulation algorithms.

The relation between the deterministic model and the stochastic counterparts was clarified in the works by Kurtz (1970, 1972). It was proved that the stochastic models converge to the deterministic ones if the initial amount of molecules is large. This was an important theoretical breakthrough in both Chemistry and Mathematics since it is appeared that deterministic and stochastic models are not independent alternative modelling frameworks. In fact, the deterministic model is an approximation of the stochastic one which is of the key importance when the system is large. Indeed, one of the practical problems of stochastic modelling is that for a system with a large amount of molecules, reactions can be so frequent that even a numerical simulation becomes computationally infeasible. Thus, the value of approximations which are easier to handle either numerically or theoretically cannot be underestimated (Schnoerr et al., 2017).

On the other hand, the deterministic approximation can lose an important information in many stochastic systems. While it usually provides a good approximation of the process mean value, it ignores completely other properties, for instance, variance, bimodality, tail behaviour, etc. Kurtz (1976) provided a second approximation which retains the stochastic nature by means of the diffusion process. The same equations had became popular in Chemistry under the name of Langevin equations due to the contribution by Gillespie (2000). These equations have been used in many works as a computational trick to speed up simulations of the original process. While this computational approach to the diffusion approximation proved to be fruitful, such interpretation hides in part the richness and the importance of the result by Kurtz (1976). Moreover, stochastic reaction networks attracted a renewed interest recently Anderson and Kurtz, 2015; Érdi and Lente, 2014, Santillán, 2014; Ullah and Wolkenhauer, 2011). New motivations come both from the application in the system biology, demonstrating the emergence of the stochastic effects at small scales, and from new theoretical investigations that allowed to extend mathematical results, previously known in the deterministic setting only, to the stochastic world (Anderson et al., 2017a, 2010; Cappelletti and Wiuf, 2016).

The goal of this communication is to review both deterministic and diffusion approximations of the CTMC and to explain their implications and the added value the diffusion approximation can provide for systems of the intermediate size. We emphasize that the results by Kurtz (1976) are constructive. It allows to give an explicit construction of the CTMC and the diffusion approximation coupled trajectories such that the uniform distance between them is controlled. To our knowledge, this fact has been never highlighted in the applied literature, while deserving to be understood better. We provide two examples in which the diffusion approximation is able to catch qualitative properties of the reaction networks that the deterministic model misses. We advocate that in the context of growing interest to the stochastic models, the diffusion approximation (or other new approximations of the same nature) has an important role in the development of the theory and deserves to be extended for new challenges opened by the applications. 


\section{Stochastic models of reaction networks and their ap- proximations}

\subsection{Reaction networks and deficiency}

A reaction network is a triple $\{\mathcal{S}, \mathcal{C}, \mathcal{R}\}$ such that

1. $\mathcal{S}=\left\{S_{1}, \cdots, S_{d}\right\}$ is the set of species of cardinality $d$ where $d$ is finite.

2. $\mathcal{C}$ is the set of complexes, consisting of some nonnegative integer linear combination of the species.

3. $\mathcal{R}$ is a finite set of ordered couples of complexes which is defined by the stoichiometric equations (2.1).

A reaction network of $K$ chemical reactions is specified by stoichiometric equations

$$
\sum_{i=1}^{d} c_{k i} S_{i} \rightarrow \sum_{i=1}^{d} c_{k i}^{\prime} S_{i}, k=1, \ldots, K
$$

meaning that the reaction consumes $\sum c_{k i} S_{i}$ to produce $\sum c_{k i}^{\prime} S_{i}$ where $c_{k i}, c_{k i}^{\prime}$ are nonnegative integers. The definition above implies the unique directed graph if the set of nodes coincides with the set of complexes. The inference of qualitative properties of the reaction network model is based on the algebraic properties of this graph, see e.g. Feliu and Wiuf (2015). We define $l_{k}=c_{k}^{\prime}-c_{k}$ as the reaction vector of the network where $c_{k}=\left[c_{k 1}, \ldots, c_{k d}\right]^{\mathrm{T}}$ and $c_{k}^{\prime}=\left[c_{k 1}^{\prime}, \ldots, c_{k d}^{\prime}\right]^{\mathrm{T}}$. They can be collected as the columns of the $d \times K$ stoichiometric matrix.

One of the most important algebraic properties of the reaction network graph is the deficiency. Let $L$ be the number of connected components (also known as linkage classes) of the reaction graph. The subspace of $\mathbb{Z}^{K}$ given by

$$
S=\operatorname{span}_{k}\left\{l_{k}\right\}
$$

is the stoichiometric subspace (with dimension $\operatorname{dim} S$ ) of the network. The number of complexes in the reaction network is given by $|\mathcal{C}|$. The deficiency of the network is defined as the integer

$$
\theta=|\mathcal{C}|-L-\operatorname{dim} S .
$$

The property of non-deficiency $(\theta=0)$ has important consequences on the dynamics of both deterministic and stochastic models (see Section 4 and Deficiency-Zero theorems Anderson et al., 2010) and Anderson and Kurtz (2015) for further details).

\subsection{Stochastic models of reaction networks}

A stochastic model of the reaction network is a Markov chain $Y(t)$ whose state space is subset of $\mathbb{N}^{d}$. The state vector $s=\left(s_{1}, \cdots, s_{d}\right)$ corresponds to the number of molecules of each species available in the system. If $s_{i} \geq c_{k i}$ for all $i \in\{1, \cdots, d\}$, the $k^{\text {th }}$ reaction of (2.1) can occur, updating the network from state $s$ to state $s+l_{k}$. The occurrences of the reactions determine the jumps of the Markov chain. The network follows the mass-action kinetics if the rate of reaction $k$ in state $s$ can be written in the form

$$
q_{s, s+l_{k}}=\frac{\lambda_{k}}{V^{\left\langle c_{k}\right\rangle-1}} \prod_{i=1}^{d}\left(\begin{array}{c}
s_{i} \\
c_{i k}
\end{array}\right)=V\left[\frac{\lambda_{k}}{\prod_{i=1}^{d} c_{i k} !} \prod_{i=1}^{d}\left(\frac{s_{i}}{V}\right)^{c_{i k}}+O\left(\frac{1}{V}\right)\right]
$$


where $\left\langle c_{k}\right\rangle=\sum_{i} c_{i k}, \lambda_{k}$ is a transition propensity for reaction $k$ and $V$ is the constant volume of the container in which the reactions take place.

The mass-action rates 2.2 allows the Markov chain models of reaction networks to satisfy the property of the density dependence (approximately). This allows to apply the approximation results given in this communication.

Definition 1. A family of continuous time Markov Chains $\left\{Y^{[V]}(t)\right\}$ indexed by parameter $V$ and with state spaces contained in $\mathbb{Z}^{d}$ is density dependent if its transition rates $q_{s, s+l}^{[V]}$ from any state $s$ to any other state $s+l$ can be written in the following form

$$
q_{s, s+l}^{[V]}=V f_{l}\left(\frac{s}{V}\right)
$$

where $f_{l}$ is a non-negative function defined on some subset of $\mathbb{R}^{d}$.

Intuitively, the necessary conditions of the density dependence are (i) the linear relation of transition rates on $V$ and (ii) the dependence on the density of the population levels rather than on the population values. Then, the argument $s / V$ in $(2.3)$ is the density associated with state $s$ and index $l$ is the vector of transitions. In case of reaction networks, the indexing parameter $V$ is the volume of the container and the process $Y^{[V]}(t)$ provides a number of molecules at time $t$. It becomes apparent from (2.3) that 2.2 has the approximately density dependent form. In fact, it is more common to rescale the number of molecules to the concentrations.

Definition 2. For the density dependent family $\left\{Y^{[V]}(t)\right\}$ we define the family of density processes $\left\{X^{[V]}(t)\right\}$ by setting for every $V$

$$
X^{[V]}(t)=\frac{Y^{[V]}(t)}{V} .
$$

Let us remark that the name density process originated from the population dynamics. In the reaction network model it represents the concentrations of the chemical species. Following the theory of point processes (Anderson and Kurtz, 2015; Brémaud, 1981) density process $X^{[V]}(t)$ can be written in two equivalent (in a sense of the probability law) forms. The first form is the stochastic differential equation

$$
d X_{t}=\sum_{l} \frac{l}{V} d M_{l}(t)
$$

where $M_{l}(t)$ counts the occurrences of those reactions whose effect is to increase $Y^{[V]}(t)$ by $l$, and hence to increase the density process $X^{[V]}(t)$ by $l / V$. The state dependent rate associated with $M_{l}(t)$ is

$$
q_{X^{[V]}(t), X^{[V]}(t)+l / n}^{[V]}=V f_{l}\left(X^{[V]}(t)\right) .
$$

The second representation of the process $X^{[V]}(t)$ is obtained by substituting counting process $M_{l}(t)$ by independent unit-rate Poisson process $N_{l}(t)$. The effect of reactions with different speed is achieved by the time change. The Poisson process implies a transformation that makes the individual time of each reaction to go 'faster' when a higher jump rate is needed and 'slower' otherwise. This leads to the following form of the process

$$
X_{*}^{[V]}(t)=X_{*}^{[V]}(0)+\sum_{l} \frac{l}{V} N_{l}\left[V \int_{0}^{t} f_{l}\left(X_{*}^{[V]}(s)\right) d s\right]
$$


where $N_{l}(t)$ is an independent unit-rate Poisson process that counts the occurrences of the events which increase $Y^{[V]}(t)$ by $l$ (or the density process $X^{[V]}(t)$ by $\frac{l}{V}$ ).

There are techniques (Érdi and Lente, 2014; Stewart, 1994) to characterize both initial transient period and long run behaviour of the Continuous Time Markov Chains (CTMC). However, in practice if the state space of the CTMC is large, an analytical treatment is not feasible and an approximation is needed. The key idea is to construct a simpler process to approximate the original CTMC when it models the interaction of large groups. We briefly describe two of such approximations below.

\subsection{Approximations}

For large values of volume $V$, the jumps of the stochastic process 2.5 become more frequent and have a smaller magnitude suggesting that corresponding trajectories can be approximated by continuous functions (so-called the fluid limit or the fluid approximations). In Ethier and Kurtz (1986); Kurtz (1970) a set of ordinary differential equations (ODE) providing the deterministic approximation of 2.5) if both volume and number of molecules are large is derived. This result is summarized below.

Approximation 1. Let $x(t)$ be a deterministic solution of the d-dimensional ODE system

$$
\dot{x}(t)=F(x(t))=\sum_{l \in C} l f_{l}(x(t))
$$

with initial condition $x(0)=x_{0}$. Let us assume that for each compact $K$ in the state space, the function $F$ is Lipshitz continuous in $K$ and that $\sum_{l}\left(|l|+|l|^{2}\right) \sup _{x \in K} f_{l}(x)<\infty$. Let $X^{[V]}(t)$ be as in (2.5) with the initial condition satisfying

$$
\lim _{V \rightarrow \infty} X^{[V]}(0)=x_{0}
$$

Fix time $T<\infty$. The density process $X^{[V]}(t)$ tends to $x(t)$ for all $t \leq T$ and

$$
\sup _{0 \leq t \leq T}\left|X^{[V]}(t)-x(t)\right|=O\left(\frac{1}{\sqrt{V}}\right)
$$

with probability one as $V \rightarrow \infty$. The constant time horizon $T$ is arbitrary, but finite.

See Ethier and Kurtz (1986); Kurtz (1970) for the proof. Let us remark that by equation (2.8), the parameter $V$ is related to the initial number of molecules and by letting $V$ to increase, the number of molecules in the system increases as well. Since $x(t)$ provides a strong (path-wise) approximation of the process $X^{[V]}(t)$, for large $V$ every trajectory remains bounded in a small interval around the deterministic function $x(t)$. In such a regimen the stochastic nature of the process $X^{[V]}(t)$ is lost, with only the mean being relevant and approximated by $x(t)$ (note that for finite $V$ the mean of $X^{[V]}(t)$ is not necessarily given by $x(t)$ (Jahnke and Huisinga, 2007)). The limit (2.7) coincides with the classical deterministic formulation of the reaction network models (Érdi and Tóth, 1989; Feinberg, 1972; Kurtz, 1972).

In a lot of cases (Érdi and Lente, 2014) the size of the system is not large enough to justify the deterministic approximation, and stochastic effects such as variance, skewness, bimodalities are to be included into the approximating model. A sharper continuous strong approximation that is able to capture stochastic fluctuations was obtained by Ethier and Kurtz (1986); Kurtz (1976) in terms of the diffusion process. 
Approximation 2. Let $X^{[V]}(t)$ be as in 2.5) and let $x(t)$ solve (2.7) with initial condition $x(0)=x_{0}$. Let $G_{*}^{[V]}(t)$ be a diffusion process with initial condition satisfying $X^{[V]}(0)=G_{*}^{[V]}(0)$ and $\lim _{V \rightarrow \infty} X^{[V]}(0)=x_{0}$ which solves the following stochastic differential equation (given in the integral form)

$$
G_{*}^{[V]}(t)=G_{*}^{[V]}(0)+\sum_{l} \frac{l}{V}\left[V \int_{0}^{t} f_{l}\left(G_{*}^{[V]}(s)\right) d s+W_{l}\left(V \int_{0}^{t} f_{l}\left(G_{*}^{[V]}(s)\right) d s\right)\right]
$$

where the $W_{l}(t)$ are independent standard Wiener processes. Let $E^{[V]} \subset \mathbb{R}$ be the smallest hyperrectangle (Cartesian product of d intervals) that contains the discrete state space of $X^{[V]}(t)$. Let $U$ be any open connected subset of $E^{[V]}$ that contains $x(t)$ for every $0 \leq t \leq T$. Let $\bar{f}_{l}=\sup _{x \in U} f_{l}(x)<\infty$ and suppose $\bar{f}_{l}=0$ except for finitely many $l$. Suppose $M>0$ satisfies both the two equations below for any $x, y \in U$

$$
\begin{aligned}
& \left|f_{l}(x)-f_{l}(y)\right| \leq M|x-y| \\
& |F(x)-F(y)| \leq M|x-y| .
\end{aligned}
$$

Let $\tau_{V}=\inf \left\{t: X^{[V]}(t) \notin U\right.$ or $\left.G_{*}^{[V]}(t) \notin U\right\}$. Note that $\mathbb{P}\left(\tau_{V}>T\right) \rightarrow 1$ for $V \rightarrow \infty$. Then for $V \rightarrow \infty$,

$$
\sup _{0 \leq t \leq \tau_{V} \wedge T}\left|X_{*}^{[V]}(t)-G_{*}^{[V]}(t)\right|=O\left(\frac{\log V}{V}\right)
$$

for any fixed time horizon $T$.

See Ethier and Kurtz (1986) for the proof and for a better estimate of the distance (2.12). The statement of Approximation 2 is quite complex, therefore, we provide some rephrasing of the main conclusion and the main assumptions.

Regarding the conclusion, Approximation 2 states that it is possible to construct coupled trajectories of the two processes $X_{*}^{[V]}(t)$ and $G_{*}^{V]}(t)$ on the same probability space (using the same random numbers) in the way that the maximum distance between them is vanishing with a rate $\frac{\log V}{V}$ when $V \rightarrow \infty$.

Regarding the assumption, some of them are technical, while others deserve to be discussed in more details. Firstly, the initial concentration is kept constant when the volume increases, the large systems with a huge number of molecules are approximated. Secondly, the assumptions on the functions $f_{l}(\cdot)$ are rather natural in the context of chemical kinetics as they prescribe that there is a finite number of reactions and none of them has an infinite speed. Finally, the approximation is only valid in any open set $U$ that is contained in $E^{[V]}$ and that contains the whole trajectory of the deterministic approximation $x(t)$. The introduction of such open set prevents both $X_{*}^{[V]}(t)$ and $G_{*}^{[V]}(t)$ from visiting the boundary of $E^{[V]}$. The concentration of each chemical species can never become negative. In some example the concentration of a species is unbounded, in other it has an upper bound. In the case it may be unlimited, the introduction of such open set is needed since the approximation will only work as far as the processes do not exceed any arbitrarily large but finite threshold (excluding explosions). Moreover, in the case if the concentrations vanish the results of Approximation 2 would not hold any more. Let us remark that when $V$ is large enough both processes will be arbitrary close to $x(t)$ with high probability and visits of the boundaries will become less frequent (and absent in the limit). For the medium-large size systems visits to boundary might still be possible and the approximation 
would fail. This is recognised as an important problem and has attracted a lot of attention in the literature (Angius et al., 2015; Leite and Williams, 2017; Schnoerr et al., 2014).

Importantly, the process $G_{*}^{[V]}(t)$ has the same law as the solution of the stochastic differential equation

$$
G^{[V]}(t)=G^{[V]}(0)+\sum_{l} \frac{l}{\sqrt{V}}\left[\sqrt{V} \int_{0}^{t} f_{l}\left(G^{[V]}(s)\right) d s+\int_{0}^{t} \sqrt{f_{l}\left(G^{[V]}(s)\right)} d W_{l}(s)\right]
$$

due to the theory of time changed Wiener integrals (Øksendal, 2003, Theorem 8.5.7).

We would like to emphasize that both $x(t)$ and $G^{[V]}(t)$ provide the strong approximations of $X^{[V]}(t)$ and are not different in this sense. The first term of 2.13 is similar to the term in the deterministic approximation (2.7), but the second one adds noise and represents the stochastic nature of the process. The approximation $G^{[V]}(t)$ preserves a random behaviour of the process and corresponds to the lower rate of the error in $(2.12)$ compared to rate 2.9 for the deterministic fluid approximation. As a result, this approximation can be applied in many cases where the deterministic one fails. A few examples are given in Section 4.

The process 2.13) is widely used to model chemical reactions and well-known in Chemistry under the name of Langevin equations (Gillespie, 2000), mainly as a trick to speed up simulations. In our opinion, the diffusion approximation result obtained by Ethier and Kurtz (1986) is not fully appreciated and deserves to be disseminated and applied more widely. Indeed, in addition to the guarantee that the laws of the processes $X^{[V]}(t)$ and $G^{[V]}(t)$ are similar, it gives the constructive procedure to generate discretized trajectories of the two processes $X_{*}^{[V]}(t)$ and $G_{*}^{[V]}(t)$ on the same probability space (i.e., with the same random numbers) that they stay close to each other trajectory by trajectory with probability one. Since such construction is not given (to out best knowledge) explicitly in any work easily accessible to non-mathematicians, we provide it in the next section.

\section{Construction of paired trajectories of CTMC and dif- fusion approximation}

The constructions of $X_{*}^{[V]}(t)$ and $G_{*}^{[V]}(t)$ are built on two preliminary steps and one key argument. Firstly, let $\tilde{N}(t)=N(t)-t$ be a compensated Poisson process with zero mean. Note that $\tilde{N}(t)$ is a martingale and equation 2.6 can be written as

$$
X_{*}^{[V]}(t)=X_{*}^{[V]}(0)+\sum_{l} \frac{l}{V}\left\{V \int_{0}^{t} f_{l}\left(X_{*}^{[V]}(s)\right) d s+\tilde{N}_{l}\left[V \int_{0}^{t} f_{l}\left(X_{*}^{[V]}(s)\right) d s\right]\right\}
$$

Secondly, notice that the sole difference between equation (3.1) and equation 2.10 is that independent compensated Poisson process $\tilde{N}_{l}(t)$ is substituted by independent Wiener process $W_{l}(t)$. The key argument is a consequence of the KMT theorem, named after Komlós et al. (1975). It states that paired trajectories of Wiener and Poisson processes can be constructed on the same probability space such that the uniform distance between them is suitably controlled. Following Ethier and Kurtz (1986) and Komlós et al. (1975) we state the following Proposition.

Proposition 1. Given a Wiener process $W(t)$, a compensated Poisson process $\tilde{N}(t)$ can be constructed on the same probability space such that for any $\beta>0$ there exist positive constants 
$\lambda, \kappa$ and $c$ such that

$$
\mathbb{P}\left(\sup _{t \leq \beta V}|\tilde{N}(t)-W(t)| \leq c \log V+x\right) \leq \kappa V^{-2} e^{-\lambda x}
$$

for any $V>1$ and $x>0$.

Given coupled trajectories of compensated Poisson process $\tilde{N}_{l}(t)$ and independent Wiener processe $W_{l}(t)$ constructed by Proposition 1, it is a (non-trivial) technical matter to show that the uniform distance between $X_{*}^{[V]}(t)$ and $G_{*}^{[V]}(t)$ fulfils equation (2.12). We start from the revisiting the construction needed to generate paired discretized sample paths of $\tilde{N}_{l}(t)$ and $W_{l}(t)$ and then we demonstrate how to build a discretization scheme for $X_{*}^{[V]}(t)$ and $G_{*}^{[V]}(t)$.

We would like to stress that a Poisson process can be seen as the partial sums of its increments and that the problem of approximating partial sums by Wiener process (strongly) has received a great attention in the literature. Strassen et al. (1967) has used the Skorohod's embedding scheme to provide the first construction. This construction, however, was shown to have not the best convergence rate (Csörgö and Révész, 1975). Instead, the new construction based on the quantile transformation of the increments of the original process was proposed by Csörgő and Révész (1975). The quantile transformation of each value, however, was insufficient, while transforming blocks of increments proved a step in the right direction. The intuitive explanation is based on the central limit theorem which states that the sum of several independent and identically distributed random variables (under some conditions) tends to be normally distributed which makes the quantile transformation close to the identity. Therefore, it was proposed by Csörgö and Révész (1975) to divide the values of process in blocks and to apply quantile transformations to sums in these blocks. The similar idea was used by Komlós et al. (1975) and further extended to the quantile transformation into the individual blocks. The construction by Komlós et al. (1975) was proved to achieve the best possible convergence rate and is provided below.

\subsection{Construction of paired Wiener and Poisson processes}

Importantly, the work by Komlós et al. (1975) proves the existence of coupled Poisson and Wiener processes and gives the construction of these processes. Precisely, given asequence of independent standard normal random variables $\left\{\bar{W}_{i}\right\}_{i=1 \cdots N}$, it is possible to construct sequence of independent standard random variables $\left\{\bar{N}_{i}\right\}_{i=1 \cdots k}$ with given distribution $F(x)$. It is also shown that the processes of the partial sums $T_{n}=\sum_{i=1}^{n} \bar{W}_{i}$ and $S_{n}=\sum_{i=1}^{n} \bar{N}_{i}$ fulfil

$$
\mathbb{P}\left(\sup _{1 \leq n \leq k}\left|S_{n}-T_{n}\right|>C \log k+x\right)<K \mathrm{e}^{-\lambda x}
$$

for any arbitrary $x, n$ and for some positive constants $C, K, \lambda$ which depend on $F$ only.

As stated above, the KMT theorem by Komlós et al. (1975) is constructive and gives an explicit algorithmic expression for the random variables $\left\{\bar{N}_{i}\right\}_{i=1 \cdots k}$ in terms of the sequence $\left\{\bar{W}_{i}\right\}_{i=1 \cdots N}$. This construction is also known as the Hungarian construction. Below we present its easily coded version allowing to simulate two discretized trajectories of Wiener process with drift and Poisson process based on the same random numbers. Note that one can equivalently generate either (i) Wiener process with drift and Poisson process or (ii) Wiener process and compensated Poisson process. The goal of the representation below is pedagogical, thus we 
focus on the most straightforward implementation of the method rather than on computational costs or a memory usage. We refer the reader to Komlós et al. (1975) for the mathematical justifications.

Let us consider the time interval $[0, n \Delta]$ and its discretization with fixed step $\Delta,\{0, \Delta, 2 \Delta, \ldots, n \Delta\}$. We specialize the KMT Theorem to the case when the random variables $\left\{\bar{N}_{i}\right\}_{i=1 \cdots n}$ are standardized Poisson increments

$$
\bar{N}_{i}=\frac{N_{l}(i \Delta)-N_{l}((i-1) \Delta)-\Delta}{\sqrt{\Delta}} .
$$

Then, Poisson process and Wiener process with drift having the same mean and variance can be obtained on the discretized time interval $[0, n \Delta]$ as

$$
\begin{aligned}
N(k \Delta) & =\sum_{i=1}^{k}\left(\sqrt{\Delta} \bar{N}_{i}+\Delta\right), \quad k=1, \ldots, n, \\
W(k \Delta) & =\sum_{i=1}^{k}\left(\sqrt{\Delta} \bar{W}_{i}+\Delta\right), \quad k=1, \ldots, n,
\end{aligned}
$$

where random variables $\left(\bar{W}_{i}\right)_{i=1}^{n}$ are distributed according to the standard normal distribution function with cumulative distribution function $\Phi$. The construction proceeds as follows. Given standardized Wiener increments $\left\{\bar{W}_{1}, \bar{W}_{2}, \ldots, \bar{W}_{n}\right\}$, we would like to find corresponding standardized Poisson increments $\left\{\bar{N}_{1}, \bar{N}_{2}, \ldots, \bar{N}_{n}\right\}$.

Without loss of generality, assume that the length of the trajectory $n$ can be written as $n=2^{K}$ where $K$ is positive integer. Following the notation of Komlós et al. (1975), we introduce the following quantities

$$
V_{j}=T_{2^{j}}, \quad V_{j, k}=T_{(k+1) 2^{j}}-T_{k 2^{j}}, \quad \tilde{V}_{q, k}=V_{q-1,2 k}-V_{q-1,2 k+1} .
$$

As Wiener increments $\left\{\bar{W}_{1}, \ldots, \bar{W}_{n}\right\}$ are already given, one can compute all of these quantities. The values of $V_{j, k}$ for all $j=0,1, \ldots, K-1$ and $k=1, \ldots, n-1$ can be written as elements of $K \times(n-1)$ dimensional matrix $\mathbb{V}$ with entries

$$
\left[\begin{array}{cccccccc}
T_{2}-T_{1} & T_{3}-T_{2} & \ldots & \ldots & \ldots & \ldots & \ldots & T_{n}-T_{n-1} \\
T_{4}-T_{2} & T_{6}-T_{4} & \ldots & \ldots & T_{n}-T_{n-2} & 0 & \ldots & 0 \\
T_{8}-T_{4} & T_{12}-T_{8} & \ldots & T_{n}-T_{n-4} & 0 & \ldots & \ldots & 0 \\
\vdots & \vdots & \vdots & \vdots & \vdots & \vdots & \vdots & \vdots \\
T_{n}-T_{n-2^{K-1}} & 0 & \ldots & \ldots & \ldots & \ldots & \ldots & 0
\end{array}\right]_{K, n-1}
$$

Using the elements of $\mathbb{V}, \tilde{V}_{q, k}$ for all $q=1,2, \ldots, K-1$ and $k=1, \ldots, n-1$ can be found as elements of $(K-1) \times\left(\frac{n}{2}-1\right)$ dimensional matrix $\tilde{\mathbb{V}}$

$$
\left[\begin{array}{cccccc}
V_{0,2}-V_{0,3} & V_{0,4}-V_{0,5} & \ldots & \ldots & \ldots & V_{0, n-2}-V_{0, n-1} \\
V_{1,2}-V_{1,3} & V_{1,4}-V_{1,5} & \ldots & V_{1, \frac{n}{2}-2}-V_{1, \frac{n}{2}-1} & \ldots & 0 \\
\vdots & \vdots & \vdots & \vdots & \vdots & \\
V_{K-2,2}-V_{K-2,3} & 0 & \ldots & \ldots & \ldots & 0
\end{array}\right]_{K-1, \frac{n}{2}-1}
$$


The matrix $\tilde{\mathbb{V}}$ can be computed by Algorithm 1

1: for $q=1,2, \ldots, K-1$ do

2: $\quad$ compute $V_{q, 2 k^{-}} V_{q, 2 k+1}$ for all $k \leq \frac{n}{2^{q}}-1$ and set elements of matrix with $k>\frac{n}{2^{q}}-1$ equal 0

3: end for

Algorithm 1: Computing elements of matrix $\tilde{\mathbb{V}}$

Similarly, let us introduce the quantities

$$
U_{j}=S_{2^{j}}, \quad U_{j, k}=S_{(k+1) 2^{j}}-S_{k 2^{j}}, \quad \tilde{U}_{q, k}=U_{q-1,2 k}-U_{q-1,2 k+1} .
$$

Note that $S_{i}$ are not yet known. In fact, the KMT computes $S_{i}$ using $U_{i}$ which are to be found using $V_{i}$. Let us define matrices $\mathbb{U}$ and $\tilde{\mathbb{U}}$ similarly to $\mathbb{V}$ and $\tilde{\mathbb{V}}$ such that the entries of $\mathbb{U}$ and $\tilde{\mathbb{U}}$ have the same structure, respectively, but in terms of Poisson increments $\bar{N}_{i}$.

Since the goal of the method is to compute Poisson increments based on Wiener increments, we rephrase our goal by saying that we aim to compute the first line $(j=0)$ of the matrix $\mathbb{U}$. Before Poisson increments can be computed the cumulative distributions function, conditional cumulative distributions function and corresponding quantile transformations should be defined as follows

$$
\begin{array}{ll}
F_{j}(x)=\mathbb{P}\left(U_{j}<x\right) & F_{q}(x \mid y)=\mathbb{P}\left(\tilde{U}_{q, 0}<x \mid U_{q_{0}}=y\right) \\
G_{j}(t)=\sup \left\{x: F_{j}(x) \leq t\right\} & G_{q}(t \mid y)=\sup \left\{x: F_{q}(x \mid y) \leq t\right\}
\end{array}
$$

Let us define $\operatorname{Poi}_{\lambda}(\mathrm{x})$ as distribution function of a Poisson r.v. with intensity $\lambda$. The cumulative distribution function $F_{j}(x)$ takes the form

$$
F_{j}(x)=\operatorname{Poi}_{2^{j} \Delta}\left(\sqrt{\Delta} x+2^{j} \Delta\right) .
$$

The conditional distribution function $F_{j}(x \mid y)$ can be calculated observing that if $A$ and $B$ are independent Poisson random variables with intensity $2^{j-1} \Delta$, then

$$
\mathbb{P}(A-B<t \mid A+B=j)= \begin{cases}0 & t<-j \\ \sum_{-j \leq i<t, i \leq j} \frac{\mathbb{P}\left(B=\frac{j-1}{2}, A=\frac{j+i}{2}\right)}{\mathbb{P}(A+B=j)} & -j \leq t \leq j \\ 1 & t>j .\end{cases}
$$

Noticing that $\tilde{U}_{j, 0}$ has the same distribution as $(A-B) / \sqrt{\Delta}$ and $U_{j}$ has the same distribution as $\left(A+B-2^{j} \Delta\right) / \sqrt{\Delta}$ leads to

$$
F_{j}(x \mid y)=\mathbb{P}\left(A-B<\sqrt{\Delta} x \mid A+B=\sqrt{\Delta} y+2^{j} \Delta\right) .
$$


Then, the elements of matrix $\mathbb{U}$ are computed by Algorithm 2

1: Compute $\bar{N}_{1}=G_{0}\left(\Phi\left(\bar{W}_{1}\right)\right)$

2: Compute the first column of $\mathbb{U}$ using $U_{j, 1}=G_{j}\left(\Phi\left(2^{-\frac{j}{2}} V_{j, 1}\right)\right)$

3: for $j, k$ such that $U_{j, k}$ is computed do

4: $\quad$ Compute $\tilde{U}_{j, k}=G_{j}\left(\Phi\left(2^{-\frac{j}{2}} \tilde{V}_{j, k}\right) \mid U_{j, k}\right)$

5: Compute

$$
\begin{aligned}
U_{j-1,2 k} & =\frac{1}{2}\left(U_{j, k}-\tilde{U}_{j, k}\right) \\
U_{j-1,2 k+1} & =\frac{1}{2}\left(U_{j, k}+\tilde{U}_{j, k}\right)
\end{aligned}
$$

6: end for when elements $U_{0, k}=N_{k+1}$ are found for all $k=1, \ldots, n-1$.

Algorithm 2: KMT algorithm

Algorithm 2 computes the elements of matrix $\mathbb{U}$ from the last line $(j=K-1)$ to the first one $(j=0)$. While the equations are explicit, the order of elements computations might not be straightforward at the first glance. Therefore, we provide a pseudo-code for the computation in Algorithm 3

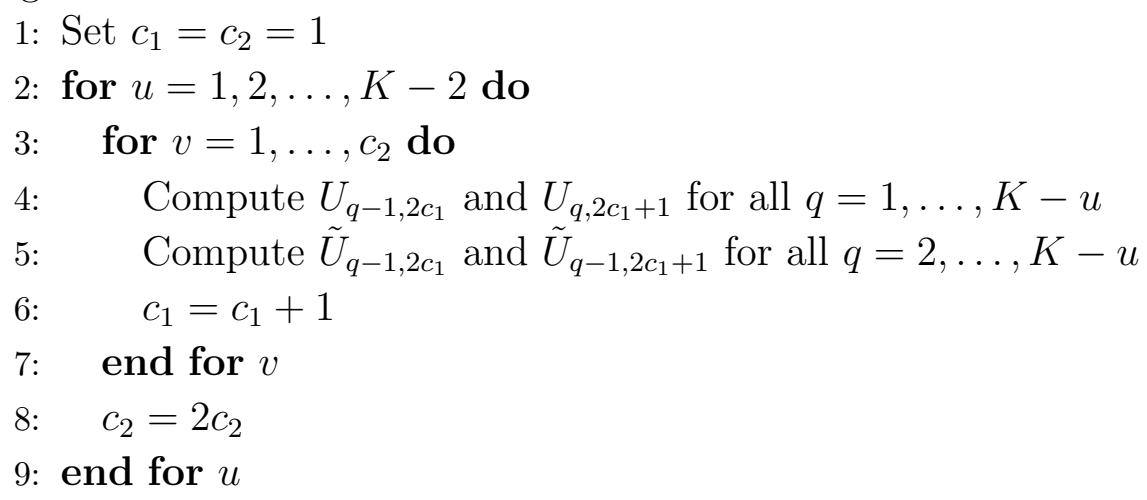

Algorithm 3: Computing elements of matrix $\mathbb{U}$

The processes needed to construct the original density dependent process $X_{*}^{[V]}(t)$ and the diffusion approximation $G_{*}^{[V]}(t)$ can be obtained by applying 3.2 and 3.3 , respectively.

\subsubsection{Illustration}

To illustrate the construction we consider a toy example with $n=16(K=4)$ and $\Delta=1$. We simulate 16 standard normal random variables $\left(\bar{W}_{i}\right)_{i=1}^{16}$ which are then truncated to

$$
\begin{aligned}
& {[-0.18,-0.93,-0.78,-1.65,-0.41,-1.10,-1.69,2.52,1.40} \\
& 0.18,-0.96,1.26,1.48,0.52,-2.25,0.47]^{\mathrm{T}}
\end{aligned}
$$

for reproducibility.

Then, $\tilde{\mathbb{V}}$ takes the form presented in Table 1 and $\mathbb{U}$ has the elements listed in Table 2 ,

\begin{tabular}{r|rrrrrrr}
\hline & $k=1$ & $k=2$ & $k=3$ & $k=4$ & $k=5$ & $k=6$ & $k=7$ \\
\hline$q=1$ & 0.87 & 0.69 & -4.21 & 1.22 & -2.23 & 0.95 & -2.72 \\
$q=2$ & -2.33 & 1.27 & 3.78 & & & & \\
$q=3$ & 1.65 & & & & & & \\
\hline
\end{tabular}

Table 1: Elements of the matrix $\tilde{\mathbb{V}}$ for the illustrative example. The missing values are zeros. 


\begin{tabular}{rrrrrrrrrrrrrrrr}
\hline$j / k$ & 1 & 2 & 3 & 4 & 5 & 6 & $\frac{7}{8}$ & 9 & 10 & 11 & 12 & 13 & 14 & 15 \\
0 & $\mathbf{- 1}$ & $\frac{-1}{-1}$ & $\frac{-1}{-1}$ & $\frac{-1}{-1}$ & $\frac{-1}{2}$ & $\frac{1}{2}$ & 2 & 0 & -1 & 1 & 2 & 0 & -1 & -1 \\
1 & $\mathbf{- 2}$ & $\frac{-2}{2}$ & $\underline{1}$ & $\frac{1}{2}$ & $\frac{-1}{-2}$ & & & & & & & & \\
2 & $\mathbf{- 1}$ & $\underline{2}$ & $\underline{0}$ & & & & & & & & & & & & \\
3 & $\mathbf{2}$ & & & & & & & & & & & & & & \\
\hline
\end{tabular}

Table 2: Elements of the matrix $\mathbb{U}$ for the illustrative example. The missing values are zeros. The bold, underlined, overlined and figures in italics correspond to the corresponding order of computing the elements.

where different fonts, under and over lines correspond to the order of computing. The procedure starts from the first column (black bold) and filling up next two columns (underlined). Then each new column is used to obtain two more columns: columns 4-7 (overlined) and 8-15 (in italics), subsequently. It is easy to see that blocks of filling doubles (1, 2, 4 and 8 columns), respectively. This fact is coded using $c_{2}$ in Algorithm 3. To compute the process of interest we apply (3.2) and (3.3). The obtained pair of processes is given in Figure 1. It is demonstrated

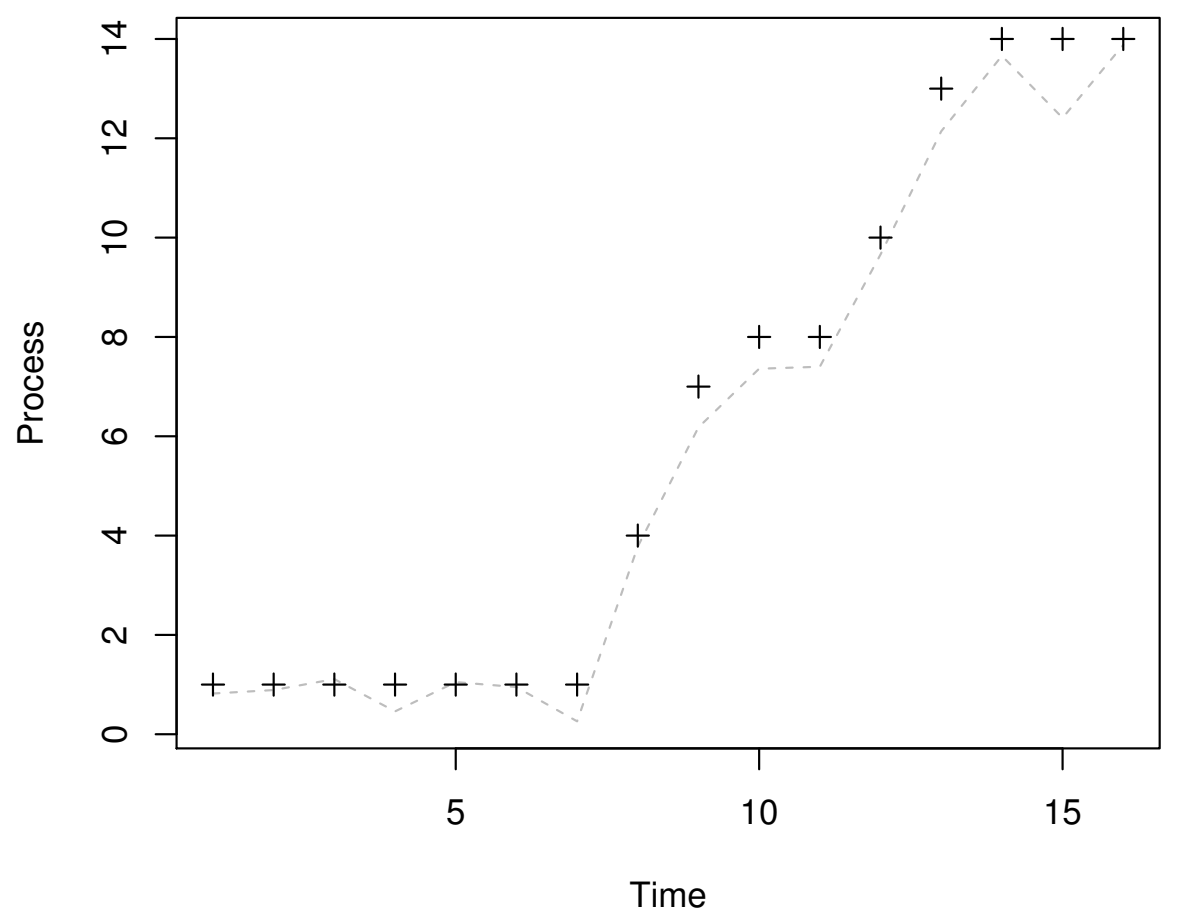

Figure 1: The pair of distretized Wiener process with drift (dashed grey line) $W_{i}$ and Poisson $N_{i}$ process (black cross)

that the constructed Poisson process captures the behaviour of the Wiener process with drift and the distance between these processes is controlled.

The explicit construction allows to provide a hint why the KMT construction has the best possible rate. As the construction fits different blocks there are two kind of errors arise: (i) the sum of the errors of the quantile transformation and (ii) the maximum of the maxima of the partial sums in the individual block. Then, the construction chooses the optimal tradeoff between these two errors. Furthermore, the simple quantile transformation $\hat{U}_{2 n}$ of $\tilde{V}_{2 n}$ is strictly independent of $U_{2 n}$ and therefore the joint distribution of $\hat{U}_{2 n}$ and $U_{2 n}$ is not equal to 
the desirable one. This problem is solved by the conditional quantile transformation which fixes the value of $U_{2 n}$.

\subsection{Paired trajectories of the CTMC and of the diffusion approxi- mation}

As the processes $N_{l}(t)$ and $W_{l}(t)$ are continuous time processes, one can compute the discretised trajectories $\hat{X}^{[V]}(t)$ of density process $X_{*}^{[V]}(t)$ given in 2.6) using an Euler scheme with step $\delta$ (which might not to coincide with step $\Delta$ in Section 3.1). We would get

$$
\begin{aligned}
\hat{X}^{[V]}(j \delta)= & \hat{X}^{[V]}((j-1) \delta)+ \\
& +\sum_{l} \frac{l}{n}\left[N_{l}\left(n \delta \sum_{k=0}^{j-1} f_{l}\left(\hat{X}^{[V]}(k \delta)\right)\right)-N_{l}\left(n \delta \sum_{k=0}^{j-2} f_{l}\left(\hat{X}^{[V]}(k \delta)\right)\right)\right],
\end{aligned}
$$

for $j=1, \ldots, N$ with $\hat{X}^{[V]}(0)=X^{[V]}(0)$. Similarly, one can obtain the discretised trajectories $\hat{G}^{[V]}(t)$ of the diffusion approximation $G_{*}{ }^{[V]}(t)$ given in 2.10 by

$$
\begin{aligned}
\hat{G}^{[V]}(j \delta)= & \hat{G}^{[V]}((j-1) \delta)+ \\
& +\sum_{l} \frac{l}{n}\left[W_{l}\left(n \delta \sum_{k=0}^{j-1} f_{l}\left(\hat{G}^{[V]}(k \delta)\right)\right)-W_{l}\left(n \delta \sum_{k=0}^{j-2} f_{l}\left(\hat{G}^{[V]}(k \delta)\right)\right)\right],
\end{aligned}
$$

for $j=1, \ldots, N$ with $\hat{G}^{[V]}(0)=G_{*}^{[V]}(0)$. Since processes $N_{l}(t)$ and $W_{l}(t)$ are not available in continuous time, but only on a discrete grid of amplitude $\Delta$, one needs to introduce a further approximation by replacing the four times

$$
\begin{array}{ll}
n \delta \sum_{k=0}^{j-1} f_{l}\left(\hat{X}^{[V]}(k \delta)\right), & n \delta \sum_{k=0}^{j-2} f_{l}\left(\hat{X}^{[V]}(k \delta)\right) \\
n \delta \sum_{k=0}^{j-1} f_{l}\left(\hat{G}^{[V]}(k \delta)\right), & n \delta \sum_{k=0}^{j-2} f_{l}\left(\hat{G}^{[V]}(k \delta)\right)
\end{array}
$$

by the closest times on the grid of obtained Wiener and Poisson processes. This would often require long trajectories of $N_{l}$ and $W_{l}$ using extremely small step $\Delta$ to get $\hat{G}^{[V]}(t), \hat{X}^{[V]}(t)$ trajectories of a moderate length. However, the challenge is computational only and can be resolved by storing long trajectories of these processes. As a final remark, we would like to emphasize that we further consider two reaction network examples for which the trajectories of CTMC and its strong diffusion approximation are provided (see Figure 4 and Figure 7). However, due to the computation costs, it is strongly recommended to a reader to simulate independent trajectories of (2.5) and (2.13) using the classical algorithms instead if the trajectory-by-trajectory behaviour is not of interest.

\section{Examples}

In this section we describe two examples of the chemical reaction systems taken from the recent literature. The aim is to discuss the ability of the deterministic and diffusion approximations to capture the dynamical properties of the original Markov Chain. 


\subsection{A toy model of metabolism and an interpretation of the defi- ciency}

The deficiency of the network has been introduced as an algebraic property of the reaction graph in Section 2.1. Polettini et al. (2015) proposed a thermodynamic interpretation of the deficiency in terms of the entropy balance. According to this interpretation, the deficiency can be understood as a number of the 'hidden' closed pathways, or thermodynamic cycles. In case $\theta=0$, the average stochastic dissipation rate equals the rate of the corresponding deterministic model. They proposed the following toy model inspired by metabolism for the illustration

$$
\begin{gathered}
\mathrm{nE} \underset{\lambda_{6}}{\stackrel{\lambda_{5}}{\rightleftharpoons}} \emptyset \underset{\lambda_{2}}{\stackrel{\lambda_{1}}{\rightleftharpoons}} \mathrm{N} \\
\mathrm{N}+\mathrm{mE} \underset{\lambda_{4}}{\stackrel{\lambda_{3}}{\rightleftharpoons}}(\mathrm{m}+\mathrm{n}) \mathrm{E}
\end{gathered}
$$

where $\mathrm{N}$ is number of nutrients and $\mathrm{E}$ is number of tokens of energy. The first reaction introduces (eliminates) nutrients and energy to (from) the environment. The second reaction processes the nutrients and $m$ tokens of energy to produce more energy and vice versa. Following Polettini et al. (2015), we fix $n=2$. The stoichiometric matrix

$$
\left[\begin{array}{cccccc}
1 & -1 & -1 & 1 & 0 & 0 \\
0 & 0 & 2 & -2 & -2 & 2
\end{array}\right]
$$

displays in the $i$-th column the increment caused in (N,E) by the reactions with propensities $\lambda_{1}, \ldots, \lambda_{6}$ in system (4.1). The approximate rates of reactions (neglecting the terms with higher order in $1 / V$ in equation (2.2) ) equal

$$
\begin{gathered}
q_{(N, E),(N+1, E)}^{(1)}=\lambda_{1} V, \quad q_{(N, E),(N-1, E)}^{(2)}=\lambda_{2} N \\
q_{(N, E),(N-1, E+2)}^{(3)}=\lambda_{3} \frac{N E^{m}}{V^{m}}, \quad q_{(N, E),(N+1, E-2)}^{(4)}=\lambda_{4} \frac{E^{2+m}}{V^{1+m}} . \\
q_{(N, E),(N, E-2)}^{(5)}=\lambda_{5} \frac{E^{2}}{V}, \quad q_{(N, E),(N, E+2)}^{(6)}=\lambda_{6} V
\end{gathered}
$$

If $m$ is strictly positive, the network is made of 5 complexes with 2 connected components and the stoichiometric space has a dimension of 2 . Then, the deficiency equals $\theta=5-2-2=1$ and is non-vanishing. In contrast, if $m=0$, the network is made of just 3 complexes, it has the single connected component and the stoichiometric space has a dimension of 2 . Thus, there is no deficiency in the system $\theta=3-1-2=0$.

Following the choice of parameters by Polettini et al. (2015), we set $\lambda_{1}=10, \lambda_{2}=1, \lambda_{3}=$ $10, \lambda_{4}=1, \lambda_{5}=10, \lambda_{6}=1$. Trajectories of the stochastic model (CTMC) of the system (4.1) in both non-deficient $(m=0)$ and deficient $(m=3)$ cases are given in Figure 2, Figure 2 also shows the deterministic approximation which solves the system of ODEs

$$
\begin{gathered}
\dot{u}=10-u-10 u e^{m}+e^{n+m} \\
\dot{e}=2\left(10 u e^{m}-e^{n+m}+1-10 e^{n}\right)
\end{gathered}
$$

where the variable $(u, e)$ are interpreted as nutrients concentrations $u=N / V$ and energy concentration $e=E / V$. We fix the value $V=600$ as in the original example. To generate 

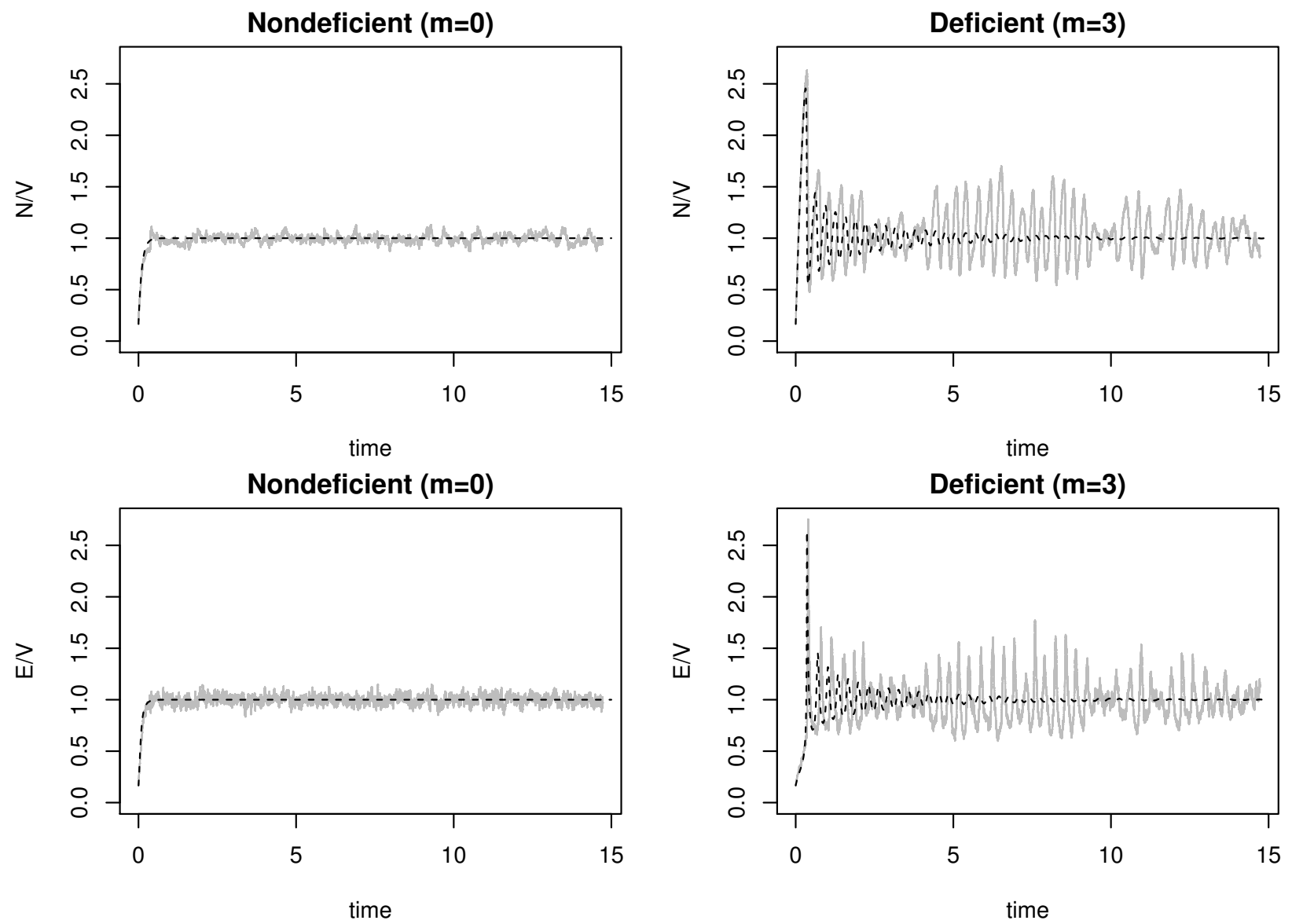

Figure 2: Deterministic (dashed black) and stochastic (solid grey) trajectories of N/V (upper part) and $\mathrm{E} / \mathrm{V}$ (lower part) in the system (4.1) in the nondeficient case (left panels, $\mathrm{m}=0$ ) and in the deficient case (right panels, $\mathrm{m}=3$ ).

the trajectory of the CTMC we use the stochastic simulation algorithm by Gillespie (1977) implemented in $\mathrm{R}$ ( $\mathrm{R}$ Core Team, 2017).

While being quite accurate in the non-deficient case, the deterministic approximation fails to catch properties of the stochastic system in the deficient case for the chosen value of $V$. Indeed, according to the deterministic model, for $m=3$ the system should display damped oscillation around the equilibrium that becomes of negligible amplitude as time goes. At the same time, the stochastic model prescribes sustained oscillations that are reducing their amplitude. This important qualitative feature is missed by the deterministic model. We remark that the inadequacy of the deterministic approximation is due to the choice of $V$. While the result in Approximation 1 says that the deterministic approximation is valid for $V$ large enough, it, however, fails to reflect the properties of the original process for the original choice of $V$.

We further investigate whether the diffusion approximation is able to capture the qualitative dynamical properties of the system. Trajectories of the diffusion approximation (2.13) are given in Figure 3 ,

One can see that oscillations are not damped according to the diffusion approximation. The trajectories of the diffusion closely resemble the behaviour of the original Markov chain. Importantly, the computation cost to compute the diffusion approximation is significantly lower than for the original CTMC. For instance, to obtain a trajectory up to $t=50$, CTMC takes 

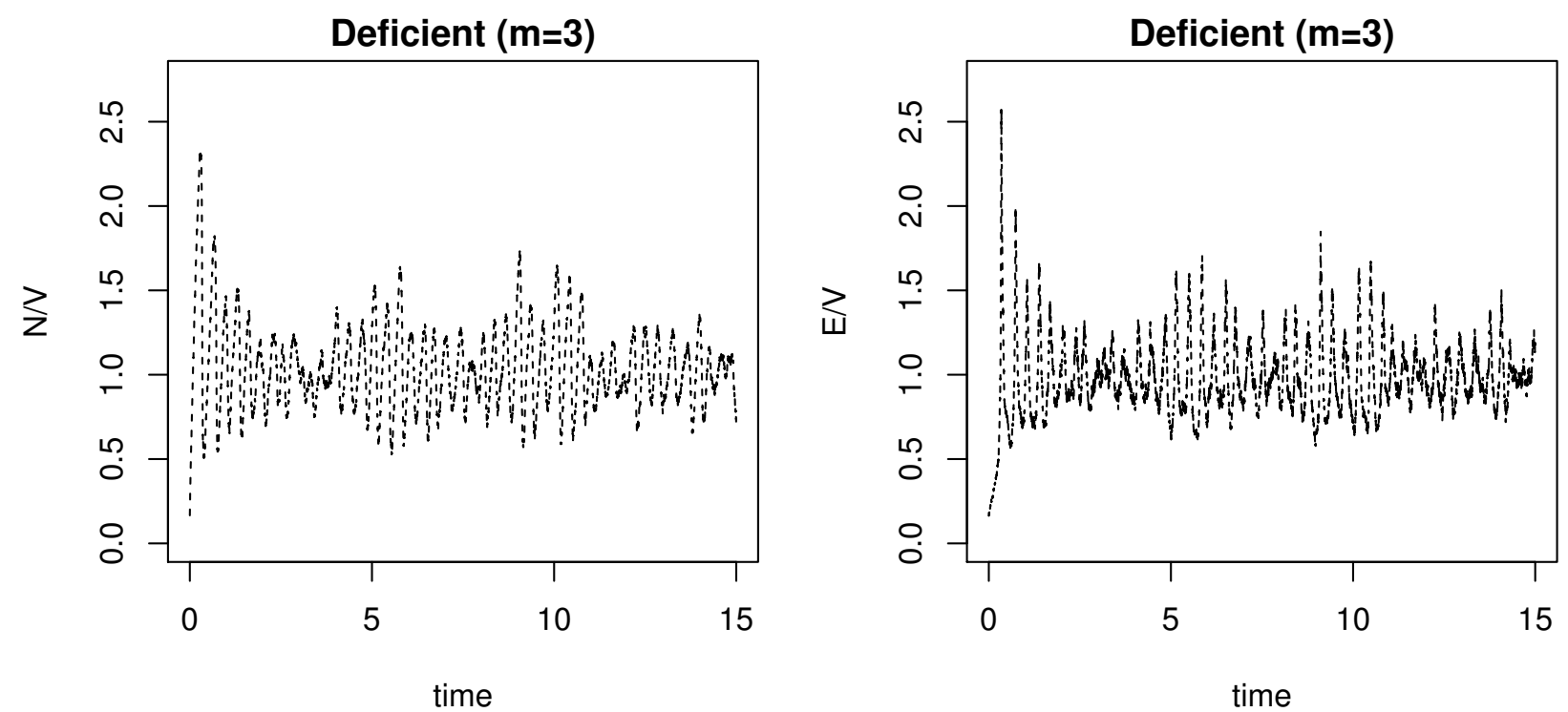

Figure 3: Trajectories of N/V (left) and E/V (right) according to the diffusion approximation (2.13), for the system (4.1) only in the deficient case $m=3$. The diffusion approximation is in very good agreement with the Markov Chain of Figure 2

nearly 14.7 seconds, while the diffusion approximation takes less than 1.8 seconds. Note, however, that trajectories in Figure 3 are generated independently of CTMC, by Euler-Maruyama discretization method, applied to the equation (2.13). To check how well the diffusion can approximate the trajectory of the CTMC given the same random numbers, we apply the algorithms described in Section 3 to generate paired trajectories. Due to the high computational costs, we limit the time to 2 which would be enough to see the general pattern. Two paired trajectories are given in Figure 4.

One can see that the corresponding trajectories are located close to each other to a very high extent and are in agreement. It follows that the diffusion approximation can mimic the behaviour of the original process, but with much less computational and analytical costs. Finally, we would like to outline that the diffusion approximation should not be considered as a short cut (to reduce the simulation time) only, as the theoretical analysis of systems with oscillations can been also performed on its basis (Baxendale and Greenwood, 2011).

\subsection{A minimal chemical reaction systems with bistability}

A bistable system is a system which has two stable equilibrium states and can be resting in either of these states. Bistable systems have been studied extensively to analyse kinetics, nonequilibrium thermodynamics and stochastic resonance. Due to its outstanding importance, the theoretical foundations of the bistability such as necessary and sufficient conditions have attracted an extensive attention in the literature, see, e.g., Joshi and Shiu (2013); Wilhelm $(2009)$.

The approach to formulate the necessary conditions of the bistability proposed by Wilhelm $(2009)$ is to find a corresponding minimal bistable chemical system (MBCS). The authors use the wording chemical system to indicate a special case of a mass-action system such that all the reaction involved are at most bimolecular. More complicated reactions are indeed believed 

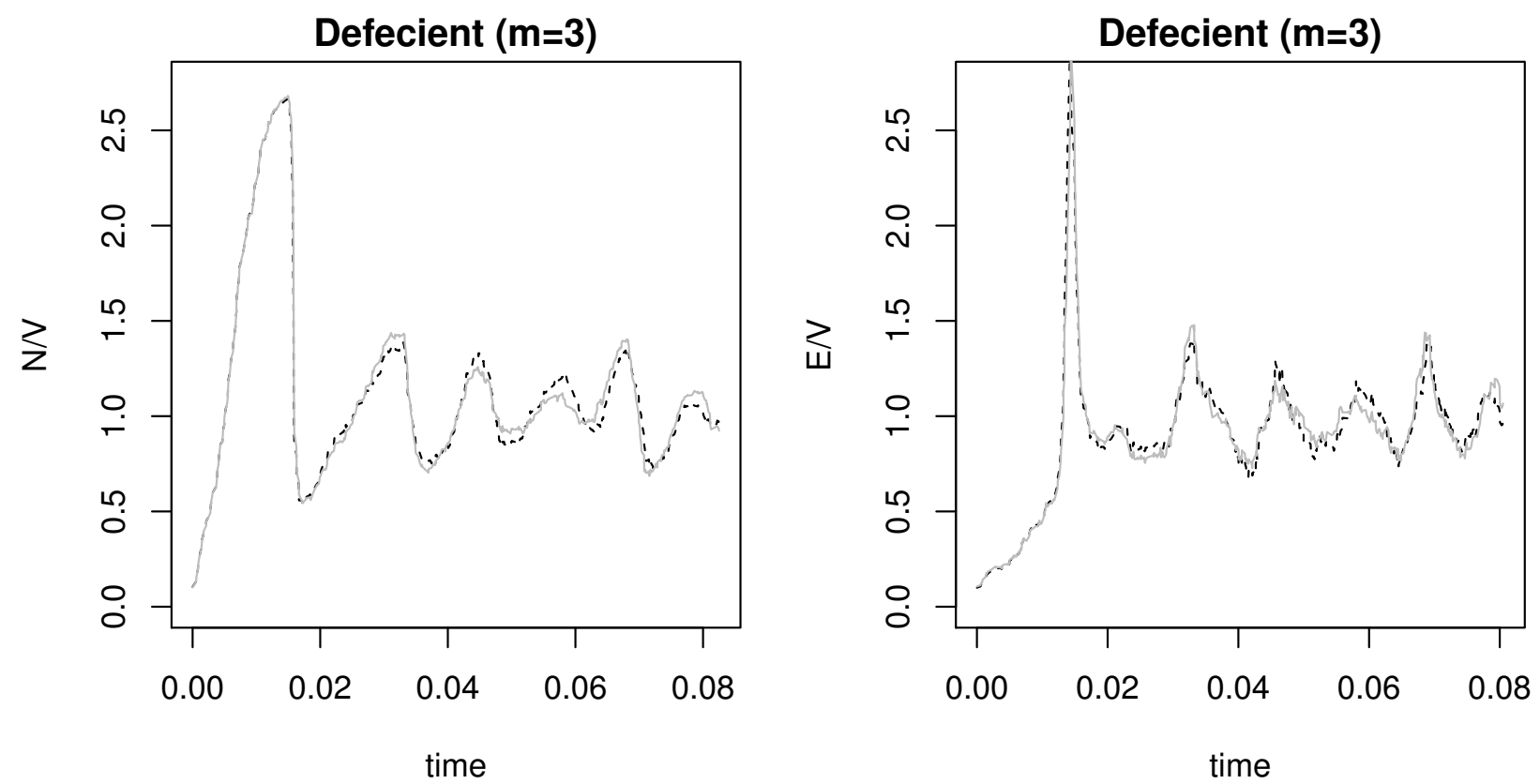

Figure 4: Paired trajectories of N/V (left) and E/V (right) according to the CTMC, (grey line) and the diffusion approximation, (dashed black line) for system (4.1) in the deficient case $m=3$.

not to be physical. We refer a reader to the original proposal of Wilhelm (2009) for the detailed definition of the minimal chemical system and for the comparison to alternative definitions, for instance, Schlogl model (Schlögl, 1972). The proposed MBCS consists of four reactions

$$
\begin{gathered}
\mathrm{S}+\mathrm{Y} \stackrel{\lambda_{1}}{\longrightarrow} 2 \mathrm{X} \\
2 \mathrm{X} \stackrel{\lambda_{2}}{\longrightarrow} \mathrm{X}+\mathrm{Y} \\
\mathrm{X}+\mathrm{Y} \stackrel{\lambda_{2}}{\longrightarrow} \mathrm{Y}+\mathrm{P} \\
\mathrm{X} \stackrel{\lambda_{4}}{\longrightarrow} \mathrm{P}
\end{gathered}
$$

where $X, Y$ are reactants and $S, P$ are substrates and products whose concentrations are kept fixed. The corresponding stoichiometric matrix takes the form

$$
\left[\begin{array}{cccc}
2 & -1 & -1 & -1 \\
-1 & 1 & 0 & 0
\end{array}\right]
$$

with the same convention adopted for equation (4.2) and the approximate rates (neglecting the terms with higher order in $1 / V$ in equation (2.2) ) are

$$
\begin{aligned}
q_{(X, Y),(X+2, Y-1)}^{(1)} & =\lambda_{1} Y, \quad q_{(X, Y),(X-1, Y+1)}^{(2)}=\lambda_{2} \frac{X^{2}}{V} \\
q_{(X, Y),(X-1, Y)}^{(3)}=\lambda_{3} \frac{X Y}{V}, & q_{(X, Y),(X-1, Y)}^{(4)}=\lambda_{4} X
\end{aligned}
$$

where the constant concentration of $S$ is incorporated in $\lambda_{1}$. The system can be described by the ODE system

$$
\dot{x}=2 \lambda_{1} y-\lambda_{2} x^{2}-\lambda_{3} x y-\lambda_{4} x
$$




$$
\dot{y}=\lambda_{2} x^{2}-\lambda_{1} y
$$

where $x$ and $y$ are concentrations of $X$ and $Y$, respectively. Setting $\lambda_{2}=1$, without restrictions of generality (Wilhelm, 2009) has shown that the system has three steady states $\bar{x}_{1}=\bar{y}_{1}=0$ and $\bar{x}_{2,3}=\frac{\lambda_{1} \pm \sqrt{\lambda_{1} D}}{2 \lambda_{3}}, \bar{y}_{2,3}=\frac{\bar{x}_{2,3}^{2}}{\lambda_{1}}$ where $D=\lambda_{1}-4 \lambda_{3} \lambda_{4}$ and the steady states 1 and 3 are stable and the steady state 2 is unstable. Following Wilhelm (2009) we set $\lambda_{1}=8, \lambda_{2}=\lambda_{3}=1$ and $\lambda_{4}=1.5$ for the illustration. In this case the steady states are $\bar{x}_{1}=\bar{y}_{1}=0, \bar{x}_{2}=2, \bar{y}_{2}=1 / 2$ and $\bar{x}_{3}=6, \bar{y}_{3}=9 / 2$. One hundred trajectories of the CTMC corresponding to system (4.2) and starting at the unstable steady state $x(0)=2, y(0)=1 / 2$ are given in Figure 5 where the deterministic approximation of the system is also presented.
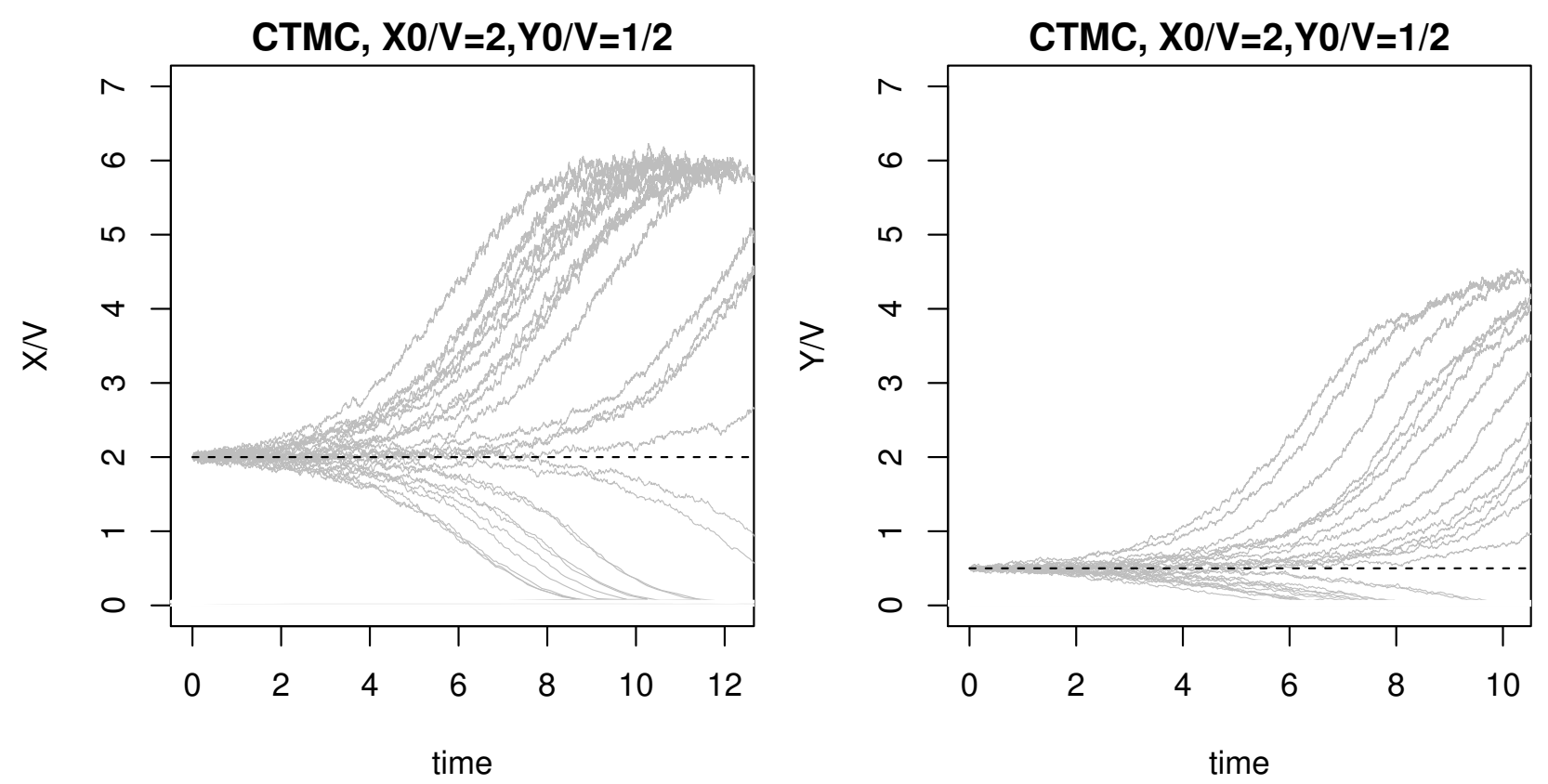

Figure 5: 100 stochastic (grey line) and 1 deterministic (black dashed line) trajectories of $X / V$ (left panel) and $Y / V$ (right panel) in system (4.2) starting at the unstable steady state $x(0)=2, y(0)=1 / 2$.

The different trajectories originating at the same unstable steady state are driven by the noise, towards one of the stable equilibria picked randomly. Let us remark that despite many trajectories are initially attracted to the upper equilibrium, sooner or later they will escape its domain of attraction and they will end up visiting the state $(0,0)$ that is absorbing. Notice that this effect cannot be illustrated in the simulations since the time required to leave the upper equilibrium is much larger than the time windows that one can explore.

The deterministic approximation is not able to capture this complex and rich behaviour of the system. Therefore, this approximation could lose important properties of the original process and should not be used. We then investigate the behaviour of the diffusion approximation. One hundred discretized trajectories (starting at the same point of unstable steady state) of the diffusion approximation are given in Figure 6.

The diffusion approximation mimics the qualitative behaviour of the original CTMC very closely. Further, we study whether the diffusion approximation is able to reproduce the behaviour of the original process for different starting points. We calculate the proportion of trajectories of the CTMC and of the diffusion approximation that is attracted to each steady 

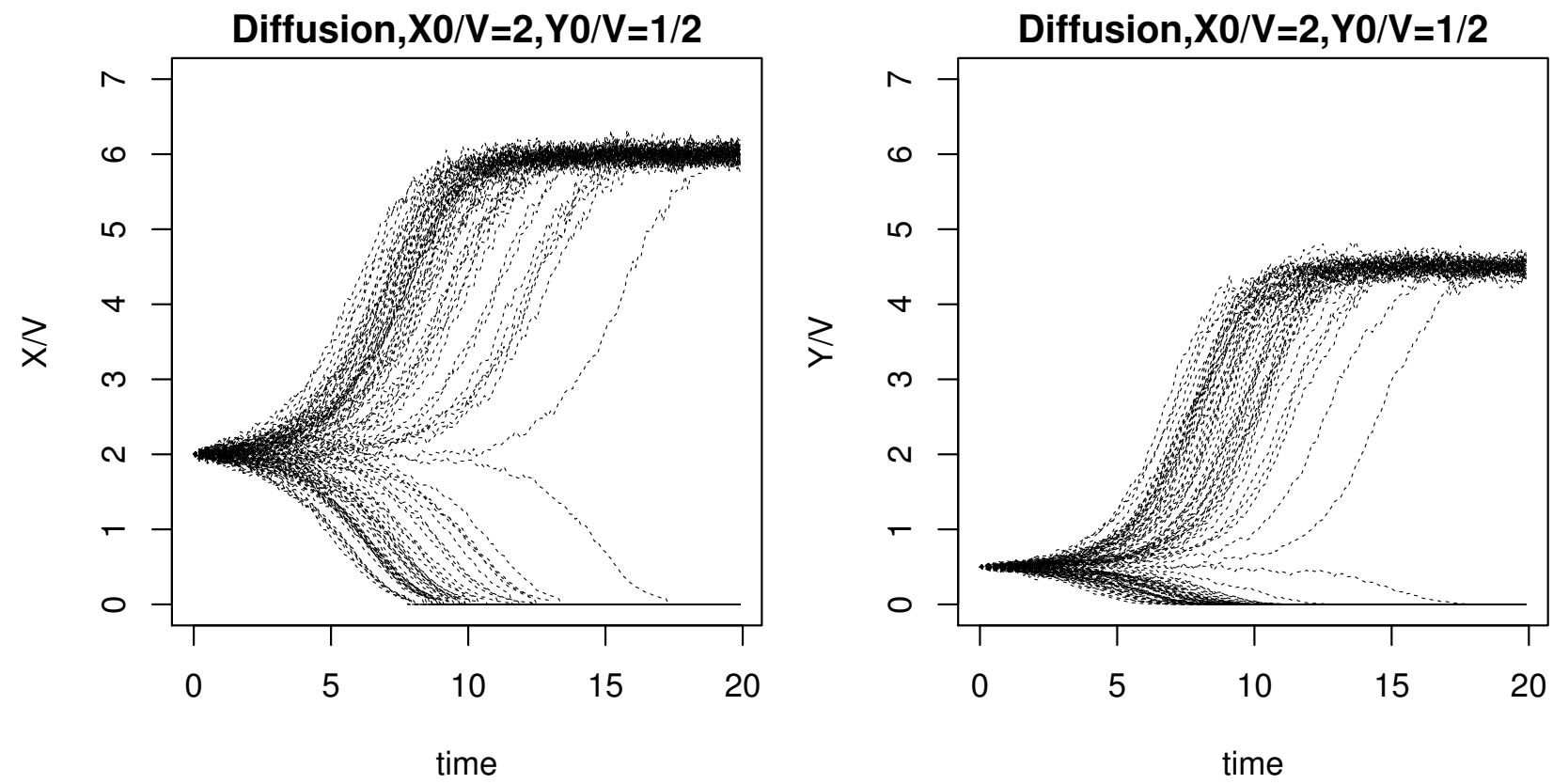

Figure 6: 100 discretized trajectories (dashed black lines) of the diffusion approximation of $X / V$ (left panel) and $Y / V$ (right panel) starting at the unstable steady state $x(0)=2, y(0)=1 / 2$.

state for different initial conditions at some fixed time point $t$. The results of nine sets of the initial points and the fixed time $t=20$ for the CTMC and the diffusion approximation are given in Table 3. Clearly, the diffusion approximation correctly reflects the behaviours of the

Table 3: The proportion of times the CTMC (upper lines) and the diffusion approximation (lower lines) is attracted to the first steady state. The results are based on $10^{4}$ replications.

\begin{tabular}{cccc}
\hline Initial point & $y(0)=0.45$ & $y(0)=0.5$ & $y(0)=0.55$ \\
$x(0)=1.95$ & $94.89 \%$ & $75.24 \%$ & $34.85 \%$ \\
& $94.89 \%$ & $75.54 \%$ & $34.49 \%$ \\
& & & \\
$x(0)=2.00$ & $85.12 \%$ & $49.63 \%$ & $14.76 \%$ \\
& $85.23 \%$ & $49.55 \%$ & $14.48 \%$ \\
& & & \\
$x(0)=2.05$ & $65.09 \%$ & $25.04 \%$ & $4.83 \%$ \\
& $65.52 \%$ & $25.45 \%$ & $4.63 \%$ \\
\hline
\end{tabular}

original process up to the moment at which the absorbing state $(0,0)$ (a boundary of the state space) is reached, as described in the comments following the statement of Approximation 2. Importantly, the computation time for 100 trajectories of the CTMC was nearly 96 minutes, while the diffusion approximation took a half of the minute. Both this and its good property to mimic the behaviour of the original process make the diffusion approximation a reasonable tool to study the behaviour of the minimal bistable chemical system.

As a further investigation on the diffusion approximation, we now compare the paired trajectories of the original process and the diffusion. Again, we limit time to $t=3.5$ due to the computational cost. The two trajectories are plotted in Figure 7. One can see that the both trajectories show a great agreement on the whole trajectory. The distance between processes 

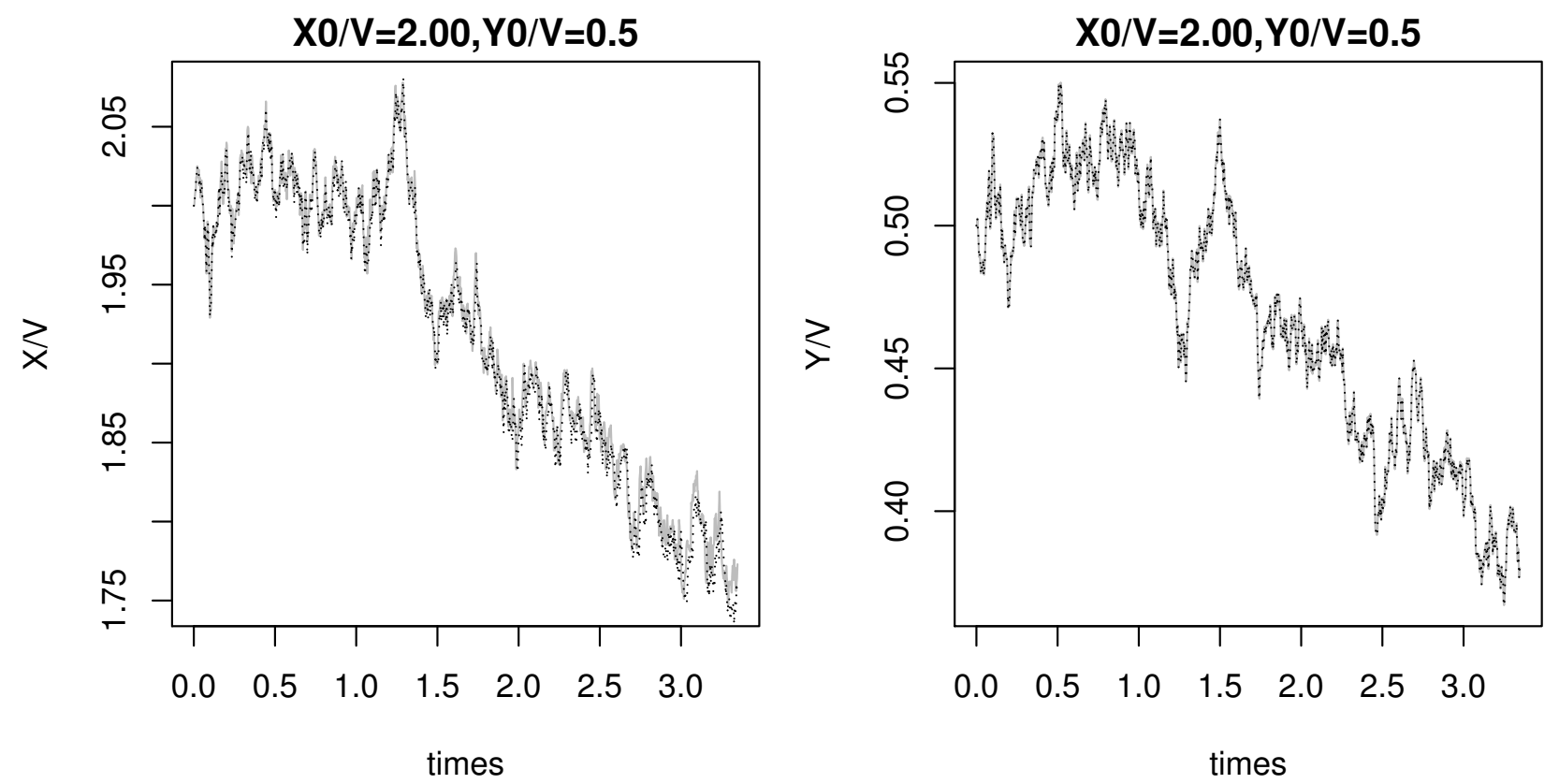

Figure 7: Paired discretized trajectories of the diffusion approximation (dashed black) and of the original CTMC (grey solid) of $X / V$ (left panel) and $Y / V$ (right panel) starting at the unstable steady state $x(0)=2, y(0)=1 / 2$.

stays little. The same conclusions were obtained for different starting values and, therefore, are not provided here.

\section{$5 \quad$ Limitations and perspective}

In this work we demonstrated that the deterministic and diffusion approximations are useful tools in the modelling of reaction networks. The diffusion approximation is able to capture the behaviour of the original process and is able to mimic trajectories of the CTMC for many different system. However, many questions of their applicability to important problems remain unanswered. Firstly, both approximations are derived on a finite time horizon. It is well known that deterministic equations may fail to catch the limiting distribution of the corresponding stochastic model when time goes to infinity as it happens in the Example presented in Section 4.2 , and for all the chemical systems with absolute concentration robustness (Anderson et al., 2014, 2017b). At the same time, they can capture such asymptotic behaviour correctly in case of the complex balanced stochastic systems (Cappelletti and Wiuf, 2016). Similar results are not yet obtained for the diffusion approximation. Secondly, both the diffusion and the deterministic approximations are known to fail when the state space of the processes is bounded and the boundaries are visited with non-negligible probability. This may be a major drawback for the medium-large size systems where the size is not large enough. Alternative approximations have been proposed for this case by Angius et al. (2015); Beccuti et al. (2014); E. Bibbona (2017); Leite and Williams (2017); Schnoerr et al. (2014), but the complete mathematical theory is still under development. 


\section{Acknowledgements}

Pavel Mozgunov and Thomas Jaki have received funding from the European Union's Horizon 2020 research and innovation programme under the Marie Sklodowska-Curie grant agreement No 633567.

\section{References}

Anderson, D., Enciso, G. and Johnston, M. (2014) Stochastic analysis of biochemical reaction networks with absolute concentration robustness. Journal of the Royal Society Interface, 11.

Anderson, D. F., Cappelletti, D., Koyama, M. and Kurtz, T. G. (2017a) Non-explosivity of stochastically modeled reaction networks that are complex balanced. arXiv preprint arXiv:1708.09356.

Anderson, D. F., Cappelletti, D. and Kurtz, T. G. (2017b) Finite time distributions of stochastically modeled chemical systems with absolute concentration robustness. SIAM Journal on Applied Dynamical Systems, 16, 1309-1339.

Anderson, D. F., Craciun, G. and Kurtz, T. G. (2010) Product-form stationary distributions for deficiency zero chemical reaction networks. Bull. Math. Biol., 72, 1947-1970.

Anderson, D. F. and Kurtz, T. G. (2015) Stochastic analysis of biochemical systems, vol. 1 of Mathematical Biosciences Institute Lecture Series. Stochastics in Biological Systems. Springer, Cham; MBI Mathematical Biosciences Institute, Ohio State University, Columbus, OH. URL: https://doi.org/10.1007/978-3-319-16895-1.

Angius, A., Balbo, G., Beccuti, M., Bibbona, E., Horvath, A. and Sirovich, R. (2015) Approximate analysis of biological systems by hybrid switching jump diffusion. Theoretical Computer Science, 587, 49-72.

Baxendale, P. H. and Greenwood, P. E. (2011) Sustained oscillations for density dependent markov processes. Journal of mathematical biology, 63, 433-457.

Beccuti, M., Bibbona, E., Horváth, A., Sirovich, R., Angius, A. and Balbo, G. (2014) Analysis of Petri net models through stochastic differential equation. In Proc. of International Conference on Application and Theory of Petri Nets and other models of concurrency (ICATPN'14). Tunis, Tunisia.

Brémaud, P. (1981) Point processes and queues. Springer-Verlag, New York-Berlin. Martingale dynamics, Springer Series in Statistics.

Cappelletti, D. and Wiuf, C. (2016) Product-form poisson-like distributions and complex balanced reaction systems. SIAM Journal on Applied Mathematics, 76, 411-432.

Csörgö, M. and Révész, P. (1975) A new method to prove strassen type laws of invariance principle. 1. Zeitschrift für Wahrscheinlichkeitstheorie und verwandte Gebiete, 31, 255-259.

E. Bibbona, R. (2017) Strong approximation of density dependent markov chains on bounded domains. ArXiv:1704.07481. 
Érdi, P. and Lente, G. (2014) Stochastic chemical kinetics. Springer Series in Synergetics. Springer, New York. URL: https://doi.org/10.1007/978-1-4939-0387-0. Theory and (mostly) systems biological applications.

Érdi, P. and Tóth, J. (1989) Mathematical models of chemical reactions. Nonlinear Science: Theory and Applications. Princeton University Press, Princeton, NJ. Theory and applications of deterministic and stochastic models.

Ethier, S. N. and Kurtz, T. G. (1986) Markov processes. Wiley Series in Probability and Mathematical Statistics: Probability and Mathematical Statistics. John Wiley \& Sons, Inc., New York. URL: http://dx.doi.org/10.1002/9780470316658. Characterization and convergence.

Feinberg, M. (1972) On chemical kinetics of a certain class. Archive for Rational Mechanics and Analysis, 46, 1-41.

Feliu, E. and Wiuf, C. (2015) Finding the positive feedback loops underlying multi-stationarity. BMC Systems Biology, 9.

Gillespie, D. T. (1977) Exact stochastic simulation of coupled chemical reactions. J. Phys. Chem., 81, 2340-2361.

- (2000) The chemical langevin equation. The Journal of Chemical Physics, 113, 297-306.

Jahnke, T. and Huisinga, W. (2007) Solving the chemical master equation for monomolecular reaction systems analytically. Journal of mathematical biology, 54, 1-26.

Joshi, B. and Shiu, A. (2013) Atoms of multistationarity in chemical reaction networks. Journal of Mathematical Chemistry, 51, 153-178.

Komlós, J., Major, P. and Tusnády, G. (1975) An approximation of partial sums of independent RV's and the sample DF. I. Z. Wahrscheinlichkeitstheorie und Verw. Gebiete, 32, 111-131.

Kurtz, T. G. (1970) Solutions of ordinary differential equations as limits of pure jump Markov processes. J. Appl. Probability, 7, 49-58.

- (1972) The relationship between stochastic and deterministic models for chemical reactions. The Journal of Chemical Physics, 57, 2976-2978.

- (1976) Limit theorems and diffusion approximations for density dependent Markov chains, 67-78. Berlin, Heidelberg: Springer Berlin Heidelberg.

Leite, S. C. and Williams, R. J. (2017) A constrained langevin approximation for chemical reaction network. Preprint available at the webpage http://www.math.ucsd.edu/ williams/ biochem/biochem.html.

Øksendal, B. (2003) Stochastic differential equations. Universitext. Springer-Verlag, Berlin, sixth edn. URL: https://doi.org/10.1007/978-3-642-14394-6. An introduction with applications.

Polettini, M., Wachtel, A. and Esposito, M. (2015) Dissipation in noisy chemical networks: The role of deficiency. The Journal of chemical physics, 143, 11B606_1. 
R Core Team (2017) R: A Language and Environment for Statistical Computing. R Foundation for Statistical Computing, Vienna, Austria. URL: https://www.R-project.org/.

Santillán, M. (2014) Chemical kinetics, stochastic processes, and irreversible thermodynamics. Lecture Notes on Mathematical Modelling in the Life Sciences. Springer, Cham. URL: https: //doi.org/10.1007/978-3-319-06689-9.

Schlögl, F. (1972) Chemical reaction models for non-equilibrium phase transitions. Zeitschrift für Physik A Hadrons and Nuclei, 253, 147-161.

Schnoerr, D., Sanguinetti, G. and Grima, R. (2014) The complex chemical langevin equation. The Journal of Chemical Physics, 141, 024103.

- (2017) Approximation and inference methods for stochastic biochemical kinetics - a tutorial review. Journal of Physics A: Mathematical and Theoretical, $\mathbf{5 0 .}$

Stewart, W. J. (1994) Introduction to the numerical solutions of Markov chains. Princeton Univ. Press.

Strassen, V. et al. (1967) Almost sure behavior of sums of independent random variables and martingales. In Proceedings of the Fifth Berkeley Symposium on Mathematical Statistics and Probability, Volume 2: Contributions to Probability Theory, Part 1. The Regents of the University of California.

Ullah, M. and Wolkenhauer, O. (2011) Stochastic approaches for systems biology. Springer, New York. URL: https://doi.org/10.1007/978-1-4614-0478-1.

Wilhelm, T. (2009) The smallest chemical reaction system with bistability. BMC systems biology, 3, 90 . 